\title{
III. Entwicklungszusammenarbeit
}

Philippe Besson

\section{OpenEdition}

\section{Journals}

Electronic version

URL: http://journals.openedition.org/sjep/988

DOI: $10.4000 /$ sjep.988

ISSN: 1663-9677

\section{Publisher}

Institut de hautes études internationales et du développement

\section{Printed version}

Date of publication: 1 janvier 1984

Number of pages: 89-111

ISSN: $1660-5926$

\section{Electronic reference}

Philippe Besson, « III. Entwicklungszusammenarbeit », Schweizerisches Jahrbuch für Entwicklungspolitik [Online], 4 | 1984, Online erschienen am: 03 Februar 2013, abgerufen am 08 September 2020. URL http://journals.openedition.org/sjep/988 ; DOI : https://doi.org/10.4000/sjep.988 


\section{ENTWICKLUNGSZUSAMMENARBEIT}

1983-1984 war die öffentliche Entwicklungshilfe wiederum ein Bereich der eidgenössischen Politik, über den ausführliche Debatten stattfanden. Ende 1983 wurde die Petition "Entwicklungshilfe ist eine Ueberlebensfrage", die von sieben Hilfswerken getragen wurde und über 200.000 Unterschriften erhielt, den Eidgenössischen Räten übergeben. Die Petition hatte zum Ziel, das Parlament dazu zu bringen, die Einbeziehung der öffentlichen Entwicklungshilfe in die Sparmassnahmen des Bundes rückgängig zu machen. Der Ständerat hat die Petition bereits Anfang 1984 geprüft und sie an den Bundesrat mit der Empfehlung weitergeleitet, sie im Rahmen des Möglichen in seinen künftigen Plänen zu berücksichtigen. Der Nationalrat hat dieses Thema in seiner Herbstsession 1984 behandelt.

Schliesslich hat der Bundesrat am 19. März 1984 seine Botschaft über die Fortführung der technischen Zusammenarbeit und der Finanzhilfe zugunsten der Entwicklungs/änder vorgelegt, die den Kammern die Annahme eines Rahmenkredits von 1,8 Mia Fr. für eine Mindestlaufzeit von drei Jahren vorschlägt. Diese Frage wurde vom Ständerat im Sommer 1984 und vom Nationalrat im Herbst 1984 diskutiert.

\section{DIE AUFWENDUNGEN FUER DIE OFFENTLICHE ENTWICKLUNGSZUSAMMENARBEIT 1983}

1983 hat die Schweiz (Bund, Kantone und Gemeinden) 574,3 Mio Fr. für die Entwicklungszusammenarbeit zur Verfügung gestellt, was $0,27 \%$ des BSP (1982:0,24\%) entspricht. Jedoch kann aufgrund des bei gewissen Beiträgen gehandhabten Zahlungsaufschubs (Zahlung durch "Notes") und der im Rahmen der OECD zur Darstellung dieses Verfahrens derzeit angewandten statistischen Methode die Rate von 0,31\% des BSP als Indikator der öffentlichen Entwicklungshilfe der Schweiz für 1983 angeführt werden.

Die Aufwendungen des Bundes allein erreichten 1983 565,9 Mio Fr., was rund 87 Franken pro Einwohner entspricht. Der Bund gibt seit 1982 in seiner Mitteilung an die OECD den Betrag der für die Verwaltung der Programme eingegangenen Ausgaben an, der im Betrag der öffentlichen Entwicklungshilfe aller Mitgliedsländer des Entwicklungshilfeausschusses (DAC) der OECD berücksichtigt wird. 1983 belief sich dieser Betrag auf rund 15 Mio Fr. Für 1984 sieht der Bundeshaushalt Aufwendungen in Höhe von 598 Mio Fr., einschliesslich 17 Mio Fr. für das Konjunkturbelebungsprogramm vor. 
Die nachstehende Tabelle zeigt die Finanzplanung für die laufende Legislaturperiode.

\section{FINANZPLANUNG 1984-1987 \\ DER ENTWICKLUNGSHILFE DES BUNDES}

(Aufwendungen in Mio Fr. und als Anteil des BSP)

\begin{tabular}{|c|c|c|c|c|c|}
\hline & 1984 & 1985 & 1986 & 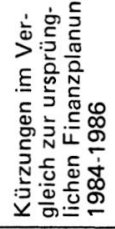 & 1987 \\
\hline $\begin{array}{l}\text { 1. Finanzplan } 1984-85 \text { vom } 5.10 .81 \\
\text { und Anträge der Departemente } \\
\text { vom Mai } 1982 \text { für } 1986\end{array}$ & $\begin{array}{c}710 \\
(0,31 \%)\end{array}$ & $\begin{array}{c}800 \\
(0,34 \%)\end{array}$ & $\begin{array}{c}968 \\
(0,39 \%)\end{array}$ & & \\
\hline $\begin{array}{l}\text { 2. Finanzplan } 1984-86 \text { nach } 10 \% \text { igen } \\
\text { linearen Kürzungen }\end{array}$ & 639 & 718 & 879 & & \\
\hline $\begin{array}{l}\text { Im Bericht vom } 4.10 .82 \text { über die } \\
\text { Finanzplanung } 1984-86 \text { vorgeschla- } \\
\text { gene Sanierungsmassnahmen in } \\
\text { Höhe von } 380 \text { Mio Fr. }\end{array}$ & -50 & -100 & -230 & -300 & \\
\hline 3. Finanzplan vom 4.10 .82 & $\begin{array}{c}589 \\
(0,26 \%)\end{array}$ & $\begin{array}{c}618 \\
(0,26 \%)\end{array}$ & $\begin{array}{c}648 \\
(0,26 \%)\end{array}$ & & \\
\hline $\begin{array}{l}\text { 4. Bundesratbeschluss vom } 16.11 .83 \\
\text { (nach } 10 \% \text { igen linearen Kürzungen) }\end{array}$ & $\begin{array}{c}581 \\
(0,26 \%)\end{array}$ & $\begin{array}{c}632 \\
(0,27 \%)\end{array}$ & $\begin{array}{c}689 \\
(0,28 \%)\end{array}$ & & $\begin{array}{c}782 \\
(0,31 \%)\end{array}$ \\
\hline $\begin{array}{l}\text { Massnahmen im Rahmen des } \\
\text { Konjunkturbelebungsprogramms }\end{array}$ & -17 & -16 & -26 & & -13 \\
\hline $\begin{array}{l}\text { 5. Finanzplan } 1985-87 \text { vom } 18.1 .84 \\
\text { (nach 10\%igen linearen Kürzungen, } \\
\text { einschliesslich Massnahmen im } \\
\text { Rahmen des Konjunkturbelebungs- } \\
\text { programms) }\end{array}$ & $\begin{array}{c}598 \\
(0,27 \%)\end{array}$ & $\begin{array}{c}648 \\
(0,28 \%)\end{array}$ & $\begin{array}{c}715 \\
(0,30 \%)\end{array}$ & -275 & $\begin{array}{c}795 \\
(0,31 \%)\end{array}$ \\
\hline
\end{tabular}

\section{Anmerkungen}

Die obigen Zahlen beziehen sich auf die öffentliche Entwicklungshilfe auf der Grundlage von Rahmenkrediten, ohne Verwaltungskosten (das heisst etwas unter den Beträgen cler Rubrik 152 der Funktionseinstufung).

Die Kürzungen im Vergleich zum ursprünglichen Finanzplan 1984-1986 geben die zu. sätzlichen zu den 10\%igen linearen Kürzungen vorgenommenen Einsparungen wieder. 
Die derzeit laufenden wichtigsten vier Rahmenkredite sind folgende :

- der am 1. April 1981 in Kraft getretene Rahmenkredit von 1.650 Mio Fr. vom 8. Dezember 1980, betreffend die Weiterführung der technischen Zusammenarbeit und der Finanzhilfe;

- der Rahmenkredit von 300 Mio Fr. vom 26. September 1979 betreffend die Beteiligung der Schweiz an der Kapitalerhöhung der Asiatischen, Interamerikanischen und Afrikanischen Entwicklungsbank, der am 1. Oktober 1979 für eine Mindestlaufzeit von vier Jahren in Kraft getreten ist;

- der Rahmenkredit von 350 Mio Fr. zur Finanzierung von wirtschaftsund handelspolitischen Massnahmen im Bereich der internationalen Entwicklungszusammenarbeit, der am 14. Dezember 1981 für die Laufzeit vom 1. Juli 1982 bis mindestens 30. Juni 1985 eröffnet wurde. Am 14. März 1983 wurde eine Erhöhung um 100 Mio Fr. angenommen, um die zusätzliche Finanzierung von Mischkrediten und Zahlungsbilanzhilfen im Rahmen des Programms zur Stärkung der schweizerischen Wirtschaft zu gewährleisten;

- der Rahmenkredit von 360 Mio Fr. betreffend die Fortführung der inter nationalen humanitären Hilfe, der am 3. Dezember 1981 für eine Laufzeit von mindestens drei Jahren ab 1. April 1982 gebilligt wurde.

Weitere Beiträge erfolgen im Rahmen der öffentlichen Entwicklungshilfe auf der Grundlage jährlicher Kreditzusagen :

- der ordentliche Beitrag der Eidgenossenschaft an das Internationale Komitee vom Roten Kreuz (IKRK) und die Beteiligung der Schweiz am Verwaltungsbudget des "Comité intergouvernemental pour les migrations" (CIM), gemäss Bundesbeschluss vom 1. Dezember 1981 bzw. vom 17. März 1954;

- die Stipendien für Studenten aus Entwicklungsländern, die an schweizerischen Universitäten studieren, gemäss Aenderung vom 7. Oktober 1983 in Bezug auf den Bundesbeschluss vom 19. Dezember 1980.

\section{Aufteilung der Kredite}

1983 betrug der Anteil der Aufwendungen des Bundes für die technische Zusammenarbeit 45,9\% (1982: 46,8\%). Der Anteil der Aufwendungen für humanitäre und Nahrungsmittelhilfe belief sich auf 21,8\% (1982: 22, $7 \%$ ), der Anteil der Finanzhilfe auf 16,8 \% (1982: 18,7\%). Die Aufwendungen für wirtschafts- und handelspolitische Massnahmen erreichten 1983 12,1\% der Gesamtaufwendungen des Bundes für die öffentliche Entwicklungshilfe (1982: 6,6\%).

Der Anteil der Geschenke im Vergleich zu den Darlehen belief sich 1983 auf $92 \%(1982: 94 \%)$. Dieser leichte Rückgang erklärt sich zum Teil aus der Zunahme der Mischkredite.

Man stellt fest, dass die am wenigsten entwickelten Länder (LLDC's) 1983 $34 \%$ der bilateralen öffentlichen Entwicklungshilfe der Schweiz erhielten 
(1982 : $36 \%$ ). Berücksichtigt man die Gruppe der am wenigsten entwickelten Länder zusammen mit den anderen einkommensschwachen Entwicklungsländern (BSP pro Kopf unter 600 Dollar), so ergibt sich, dass sie $66 \%$ der Aufwendungen erhielten (1982:67\%).

Der Anteil der bilateralen Hilfe an den Aufwendungen der öffentlichen Entwicklungszusammenarbeit nahm 1983 weiterhin zu und erreichte $74 \%$ (1982 : 70,7\%). Dieser Prozentsatz wird auch in den kommenden Jahren noch hoch sein, da die Zahlungen im Bereich der multilateralen Hilfe aufgrund der Einführung der "Notes" zum Teil aufgeschoben werden. So betrug der Anteil der multilateralen Hilfe an den Gesamtausgaben der öffentlichen Entwicklungshilfe 1983 lediglich $26 \%$.

Die geographische Aufteilung zeigt, dass Afrika weiterhin Schwerpunkt der schweizerischen Entwicklungszusammenarbeit bleibt. Die Trockenheit in den Ländern der Sahelzone ist auch derzeit äusserst besorgniserregend, so dass die Schweiz in Zusammenarbeit mit dem Ständigen Zwischenstaatlichen Komitee zur Bekämpfung der Dürre in der Sahelzone (CILSS) einen wesentlichen Teil ihrer Mittel für die Lösung dieses Problems einsetzt.

Abschliessend sei vermerkt, dass sich die Aufteilung der Hilfe wenig verändert hat, mit Ausnahme der Erhöhung der Mittel für wirtschaftliche und finanzielle Massnahmen, die jedoch lediglich konjunkturbedingt sein könnte.

\section{WESENTLICHE PUNKTE DER PARLAMENTSDEBATTEN UEBER DIE ENTWICKLUNGSZUSAMMENARBEIT}

Anlässlich der Debatten in den Räten über das Budget und das Sparprogramm stellten zwei Nationalrätinnen dem Bundesrat Anfragen über die Kürzungen der Bundesmittel für öffentliche Entwicklungshilfe und deren Auswirkungen. Frau Gurtner (Poch, BE) sprach sich gegen diese Sparmassnahmen sowie gegen die geringe Beachtung, die der Petition der Hilfswerke zuteil wurde, aus.

Frau Blunschy (CVP, SZ) bedauerte die bei der öffentlichen Entwicklungshilfe vorgenommenen Kürzungen sowie die Tatsache, dass der Nationalrat nicht beschlossen hat, die Petition der Hilfswerke zu gleicher Zeit wie das Sparprogramm zu behandeln.

Zur Erinnerung sei vermerkt, dass die öffentliche Entwicklungshilfe des Bundes für 1984 im Rahmen der im Bericht über die Finanzplanung 19841986 vom 4. Oktober 1982 vorgeschlagenen Sanierungsmassnahmen um 50 Mio Fr. und aufgrund der linearen Kürzungen gemäss Bundesratsbeschluss vom 16. November 1983 um 8 Mio Fr. reduziert wurde.

Die sozialistische Fraktion im Nationalrat brachte im Juni 1983 ein Postulat 
ein, in dem der Bundesrat aufgefordert wurde, unter anderem die Möglichkeit zu prüfen, Nicaragua im Rahmen der technischen Zusammenarbeit und der humanitären Hilfe der Eidgenossenschaft mehr Unterstützung zu gewähren. Der Bundesrat hat sich bereit erklärt, das Postulat in der Hinsicht anzunehmen, Nicaragua die gleiche humanitäre Hilfe zukommen zu lassen, wie er sie anderen Ländern gewährt.

Nationalrat Müller (EVP, AG) reichte im Juni 1983 ein Postulat ein, in dem eine Erläuterung der Kriterien, nach denen die Entwicklungshilfeprojekte des Bundes evaluiert werden, beantragt wird. Der Bundesrat hat das Postulat angenommen und seine Absicht erklärt, die Antwort in Form eines Rechenschaftsberichts vorzulegen.

Nationalrat Ruffy (SP, Vd) brachte am 16. Dezember 1983 eine Interpellation ein, in der der Bundesrat aufgefordert wird, für das brasilianische "Nordeste"-Gebiet eine erhöhte Hilfe zu gewähren. Der Bundesrat erklärte in seiner Antwort, dass für den "Nordeste" 1 Mio Fr. bereitgestellt worden seien; er fügte hinzu, dass er sich der Notlage dieses Gebietes bewusst sei und bereit sei, die Anträge von Hilfswerken im humanitären Bereich zu prüfen.

Nationalrätin Blunschy (CVP, SZ) legte am 12. März 1984 eine Interpellation vor, in der vorgeschlagen wird, angesichts des Hungers in der Welt die humanitäre Hilfe der Schweiz zu erhöhen. Frau Blunschy ist der Ansicht, dass die Situation in Afrika und im Nordosten Brasiliens besonders besorgniserregend ist.

Der Bundesrat stellte in seiner Antwort fest, dass seiner Ansicht nach die Hungersnot in den meisten Fällen auf Mängel bei der Infrastruktur und der Agrarpolitik zurückzuführen ist und er es daher vorziehe, der Entwicklungszusammenarbeit Vorrang einzuräumen. Nichtsdestoweniger wurde für Afrika ein Sonderkredit in Höhe von 5 Mio Fr. in Form von Nahrungsmittelhilfe aufgebracht. $54 \%$ der gesamten Nahrungsmittelhilfe gehen an Afrika.

Am 19. März 1984 brachte Nationalrätin Friedli (SP, Jura) eine gewöhnliche Anfrage betreffend den Manantali-Staudamm (Mali) ein.

Im November 1983 hatte der Bundesrat dem mit einem internationalen Konsortium assoziierten Unternehmen Losinger AG die Exportrisikogarantie für den Bau eines Staudamms am Senegalfluss in Manantali, Mali, einem der am wenigsten entwickelten Länder Afrikas, gewährt.

Gemäss einer für die amerikanische Regierung durchgeführten Studie wird der Bau dieses Staudamms die Umsiedlung von 10-15.000 Einwohnen auf wasserlose Böden, die nicht bewirtschaftet werden können, zur Folge haben und könnte weitere 100-300.000 Einwohner, die derzeit von den Fluten des Senegalflusses leben, ihrer traditionellen Ressourcen berauben. Die Weltbank verzichtete auf die Finanzierung dieses Projektes, das von gewissen Experten scharf kritisiert wird. 
Frau Friedli stellte dem Bundesrat die Frage, ob er zur Einhaltung des Gesetzes über die Exportrisikogarantie Einzelheiten über die erfolgte Anhörung der betreffenden Dienststellen bekanntgeben könne und ob Massnahmen zur Behebung der negativen Auswirkungen des Projekts (Wiederaufforstung, Bewässerungsplanung usw.) getroffen worden seien.

Der Bundesrat stellte in seiner Antwort fest, dass die ERG nur den schweizerischen Anteil des Projekts (29\%) decke. Die Beteiligung der Schweiz beschränkt sich auf die Ausführung des Staudamms und es besteht keine Möglichkeit der Einflussnahme auf das Projekt. Die Finanzierung war zum Zeitpunkt der Ausschreibung voll gewährleistet. Der Bundesrat ist der Ansicht, dass nur die Grundprinzipien der schweizerischen Entwicklungshilfepolitik bei der ERG zur Anwendung kommen. Von Seiten der Schweiz ist grundsätzlich nicht vorgesehen, Entwicklungsprojekte im Staudammgebiet zu unterstützen.

\section{Beratende Kommission für die internationale Entwicklungszusammenarbeit}

Diese Kommission hat die Funktion, den Bundesrat in Fragen der internationalen Entwicklungshilfe zu beraten. Sie setzt sich aus 17 Mitgliedern zusammen, die von ausserhalb der Bundesverwaltung stammen, Parlamentarier und Vertreter der Hilfswerke, der Privatwirtschaft, der Gewerkschaften, der Universitäten und der Presse. Das Sekretariat der Kommission wird von der Abteilung Politik und Forschung der DEH geführt. Die Kommission tagt viermal im Jahr.

Im Februar 1983 hat die Kommission die Vorlage eines nationalen Forschungsprogramms über die Beziehungen zwischen der Schweiz und der Dritten Welt untersucht und die Wahl dieses Themas und seine Finanzierung durch den Nationalfonds empfohlen. Ferner hat die Kommission den neuen Rahmenkredit für die Beteiligung der Schweiz am Kapital der regionalen Entwicklungsbanken einer Prüfung unterzogen.

Auf ihrer Sitzung im Mai 1983 hat sich die Kommission mit dem Haushaltsentwurf 1984 der öffentlichen Entwicklungshilfe sowie mit der Vorbereitung der 6. Konferenz der Vereinten Nationen über Handel und Entwicklung (UNCTAD VI) beschäftigt. Das letztere Thema wurde zusammen mit der beratenden Kommission für die Handelspolitik behandelt.

Auf der Tagesordnung der Sitzung vom September des gleichen Jahres stand das Entwicklungshilfeprogramm der DEH in Tansania. Die Kommission wurde über die Aktionsmöglichkeiten der Schweiz angesichts einer sehr schwierigen sozialen und wirtschaftlichen Lage informiert. Die geographische und sektorielle Schwerpunktpolitik der DEH konnte somit anhand dieses Beispiels diskutiert werden.

Auf der letzten Sitzung der Kommission (November 1983) sprachen sich die Mitglieder über die Botschaft betreffend die Weiterführung der technischen Zusammenarbeit und der Finanzhilfe zugunsten der Entwicklungs- 
länder aus. Der Kommission war ein detaillierter Plan unterbreitet worden, und zahlreiche auf der Sitzung vorgebrachte Anregungen konnten bei der endgültigen Abfassung der Botschaft berücksichtigt werden.

Die Kommission wird ferner über die wichtigsten internationalen Konferenzen und Verhandlungen auf dem laufenden gehalten, an denen die Schweiz teilnimmt, und wird über die wesentlichen Ereignisse im Bereich der Entwicklungspolitik informiert.

\section{WIRTSCHAFTS- UND HANDELSPOLITISCHE MASSNAHMEN}

Die im Bundeshaushalt aufgeführten wirtschafts- und handelspolitischen Massnahmen haben 1984 erstmals 100 Mio Fr. überschritten. Ihr Anteil an der öffentlichen Entwicklungshilfe hat sich in den letzten Jahren verfünffacht. Während im Budget 1983 noch 73,1 Mio Fr. vorgesehen waren, erreicht der Betrag 1984 aufgrund der Annahme des ersten Massnahmenpakets zur Stärkung der schweizerischen Wirtschaft 101,8 Mio Fr.

Die vom Bundesamt für Aussenwirtschaft (BAWI) verwalteten Mittel sind hauptsächlich für Zahlungsbilanzhilfen und Mischkredite bestimmt. Letztere werden in Form von gebundener Hilfe gewährt und können nur zur Versorgung mit schweizerischen Produkten verwendet werden. Ein Teil des Kredits, der bis zu $50 \%$ der Gesamtsumme betragen kann, wird vom Bund gemäss den Normen der öffentlichen Entwicklungshilfe gewährt, während der Restbetrag von Handelsbanken zur Verfügung gestellt wird.

Zum gegenwärtigen Zeitpunkt sind Kreditabkommen mit Aegypten, Honduras, Indien, Kenia, Kamerun, Marokko, dem Senegal, Sri Lanka, Thailand, Tunesien und Simbabwe abgeschlossen; mit China und Indonesien werden Verhandlungen geführt.

Ein Mischkreditabkommen über einen Betrag von 20 Mio Fr., von dem der Bund die Hälfte übernommen hat, wurde am 22. Juni 1984 mit der Westafrikanischen Entwicklungsbank abgeschlossen. Es ist das erste Mal, dass die Schweiz einer internationalen Organisation einen Mischkredit einräumt.

Die Evaluation des ersten Mischkredits an Aegypten wurde 1983 durchgeführt. Ihre Ergebnisse sind nicht öffentlich, doch sei vermerkt, dass es sich hierbei um einen Erstfall handelt.

\section{Untersuchungen über die Förderung der Importe}

1983 wurden zwei Studien über die Möglichkeiten, das Volumen der Einfuhren aus der Dritten Welt zu steigern, durchgeführt. Die eine Studie wurde vom BAWI finanziert und betraf den kommerziellen Vertrieb von Juteerzeugnissen aus Bangladesh; die andere wurde für das Aktionskomitee 
"Brot für Brüder" durchgeführt und untersuchte die Möglichkeit der Einführung eines Gütezeichens, das die Qualität des Produkts nach entwicklungspolitischen Normen garantiert.

Jute ist das Hauptausfuhrprodukt von Bangladesh (50\% der Ausfuhren). Es besteht jedoch eine starke Konkurrenz seitens der synthetischen Produkte. Die Lösung wäre eine Steigerung der Produktivität der verarbeitenden Industrie, eine allgemeine Absatzförderung und der Abschluss bilateraler Preisabkommen. Die Aussichten auf dem Schweizer Markt sind nicht sehr gut und hängen stark von der Mode und von den Erzeugnissen, je nach Verarbeitung, Form usw. ab. Obwohl der Umsatz nicht sehr hoch werden kann, könnten die Auswirkungen für einige Hersteller in Bangladesh entscheidend sein. Zum anderen besteht die Möglichkeit, das Vertriebsnetz beträchtlich zu erweitern.

Was die den Produkten der Dritten Welt vorbehaltene Gütemarke betrifft, so kann sie solange nicht eingeführt werden, wie die Herkunft der Erzeugnisse nicht vorgeschriebenermassen angegeben wird und nicht eine ausreichende Anzahl von Artikeln angeboten werden kann, was in Anbetracht der vorzunehmenden Kontrollen betreffend die Arbeits- und Produktionsbedingungen schwierig ist.

\section{Zahlungsbilanzhilfe}

In der Zeit vom Juli 1983 bis Juni 1984 erhielten die drei afrikanischen Länder Ghana, Madagaskar und Sudan von der Schweiz Zahlungsbilanzhilfen zugesprochen. Die zwei letzteren Länder hatten bereits früher solche Hilfeleistungen erhalten.

Mit dem Sudan kamen zwei Abkommen im Juli 1983 und im Mai 1984, in Höhe von jeweils 10 Mio Fr. zustande. Das erste Abkommen sieht die Finanzierung von Einfuhren von Basismedikamenten sowie Rohstoffen, Halbfabrikaten und Ersatzteilen für die Industrie und Landwirtschaft vor. Dem Sudan soll geholfen werden, seine bestehenden Produktionskapazitäten besser zu nutzen und die Versorgung mit pharmazeutischen Produkten zu verbessern.

Das zweite Zahlungsbilanzhilfeabkommen ist Teil eines mehrheitlich von der Internationalen Entwicklungsagentur (IDA) finanzierten Programms. Ziel des Programms ist die Unterstützung der Bewässerung in der Landwirtschaft zur Förderung der Baumwollproduktion. Die Kosten des Programms belaufen sich auf 90 Mio Dollar; davon entfallen 50 Mio Dollar auf die IDA.

Ein weiteres Zahlungsbilanzhilfeabkommen wurde am 12. Januar 1984 mit Madagaskar abgeschlossen. Ein Kredit von 10 Mio Fr. ist für die Finanzierung von Rohstoffen, Ersatzteilen, Halbfabrikaten und Werkzeugen für die wichtigsten Wirtschaftszweige bestimmt. Die Hilfe soll insbesondere dazu beitragen, die Reisproduktion zu steigern und die bestehenden Produktionskapazitäten im handwerklichen und industriellen Sektor besser zu nützen. 
Die Finanzierung dieser Hilfe erfolgt aus dem zusätzlichen Kredit betreffend die "Massnahmen zur Stärkung der schweizerischen Wirtschaft", die vom Bundesrat Anfang 1983 beschlossen wurden.

Auch die Zahlungsbilanzhilfe an Ghana erfolgt im Rahmen des Programms zur Belebung der schweizerischen Wirtschaft. Ihr Betrag von 12,7 Mio Fr. ist für die Sanierung des Lastwagenparks der staatlichen Transportgesellschaft Ghanas (State Transport Corporation) vorgesehen. Ueber zwei Drittel der rund 190 Last- und Zisternenwagen (der Marke Saurer) waren vor allem wegen mangelnder Ersatzteile nicht mehr fahrtüchtig.

Die Ghana zugesprochene Hilfe dient dem Kauf von notwendigen Ersatzteilen und anderem Material. Andererseits sollen daraus die von der Firma Saurer durchzuführenden Reparatur- und Unterhaltsarbeiten, sowie die Weiterbildung von Fahrern und Mechanikern finanziert werden.

Obwohl die Zahlungsbilanzhilfen zum grössten Teil nicht an den Kauf von schweizerischem Material gebunden waren, wurden die Kredite trotzdem vor allem in der Schweiz ausgegeben. 1983 gab der Sudan rund drei Viertel der erhaltenen Hilfe (10 Mio Fr.) für den Erwerb schweizerischer Lieferungen aus. Von dem Madagaskar 1982 gewährten ersten Kredit, der sich ebenfalls auf 10 Mio Fr. belief, flossen rund zwei Drittel wieder in die Schweiz zurück. Die zweite Zahlungsbilanzhilfe an Madagaskar wird vermutlich in einem noch grösseren Ausmass zu Käufen in der Schweiz führen. Was die Hilfe an Ghana betrifft, so sieht man vor, dass der Anteil der in der Schweiz auszugebenden Beträge noch höher sein wird.

\section{TECHNISCHE ZUSAMMENARBEIT UND FINANZHILFE}

Die Aufwendungen für die 1983 aufgebrachte bilaterale technische Hilfe wurden durch den 1980 bewilligten Rahmenkredit von 1.650 Mio Fr. finanziert. Dabei lag das Schwergewicht weiterhin bei der landwirtschaftlichen Entwicklung mit $27 \%$ der aufgegliederten Ausgaben. Der Anteil der Erziehung betrug $11 \%$, desgleichen jener für Banken, Handel und Tourismus, während Industrie und Handwerk einerseits und das Gesundheitswesen andererseits jeweils $9 \%$ der Mittel erhielten.

Der laufende Rahmenkredit, dessen Mittel am 31. Oktober 1984 aufgebraucht sein werden, wird durch den neuen Kredit von 1.800 Mio Fr. mit einer Laufzeit von mindestens drei Jahren ersetzt.

1983 war die DEH für 514 laufende Projekte verantwortlich (464 Projekte technischer Hilfe, davon 46 neue Aktionen, und 50 Finanzhilfeprojekte, davon 29 neue Vorhaben). 
Der der DEH für die Verwaltung zugebilligte Mitarbeiterstab blieb 1983 zahlenmässig unverändert; 143 der 144,5 genehmigten Stellen waren besetzt. Hingegen ging die Zahl der Mitarbeiter, die aufgrund eines privatrechtlichen Vertrages für Aufgaben im Ausland verpflichtet wurden, von 343 im Jahre 1982 auf 290 im Jahre 1983 zurück. In diesen Zahlen sind diejenigen Mitarbeiter nicht berücksichtigt, die in Projekten verpflichtet sind, welche von der DEH auf Regiebasis vergeben werden, für die sie jedoch selbst die Verantwortung behält. Der Rückgang der Zahl der Entwicklungshelfer hat zwei Hauptursachen : einerseits die Uebertragung von Projekten der DEH auf andere Organisationen und andererseits die Verlängerung der Vertragsfristen, wodurch die Anzahl doppelt aufgeführten Mitarbeiter verringert wird.

\subsection{Bilaterale Entwicklungszusammenarbeit}

Die bilaterale Entwicklungshilfe des Bundes belief sich 1983 auf 287,2 Mio Fr., wovon 107,5 Mio Fr. auf die technische Zusammenarbeit entfielen. Die 1983 von der DEH direkt durchgeführten Aktionen der technischen Zusammenarbeit und Finanzhilfe machten zusammengenommen 43,9\% der Gesamtaufwendungen aus. $19,7 \%$ der 287,2 Mio Fr. (1982 : 15,5\%) wurden an schweizerische Institutionen und Unternehmen zur Durchführung dieser Aktionen auf Regiebasis vergeben.

\section{GEOGRAPHISCHE AUFTEILUNG DER BILATERALEN AKTIONEN DER DEH IM BEREICH DER TECHNISCHEN ZUSAMMENARBEIT UND DER FINANZHILFE FUER 1983}

(Auszahlungen in Mio Fr. und Prozent)

\begin{tabular}{lrr} 
Gebiet & Mio Fr. & \multicolumn{1}{c}{$\%$} \\
\hline Afrika & 118,1 & 41,1 \\
Lateinamerika & 52,0 & 18,1 \\
Asien & 95,3 & 33,2 \\
Europa & 1,0 & 0,3 \\
Nicht aufgegliedert & 20,8 & 7,3 \\
INSGESAMT & $\mathbf{2 8 7 , 2}$ & $\mathbf{1 0 0 , 0}$
\end{tabular}

\section{Anmerkung}

Innerhalb der Gruppe der ärmsten Länder ging der grösste Teil der Hilfe an 18 sogenannte "Schwerpunktländer" und ein Schwerpunktgebiet, die insgesamt $60 \%$ der schweizerischen bilateralen Hilfe erhielten. 


\section{Zusammenarbeit mit schweizerischen Hilfswerken}

Eine Reihe schweizerischer Hilfswerke übten ihre Entwicklungshilfetätigkeit bereits vor der DEH aus. Sie haben zahlreiche Projekte in den Entwicklungsländern durchgeführt und dadurch weitreichende Erfahrung erlangt. Als Privatorganisationen können sie in manchen Fällen besser als die öffentlichen Institutionen die gesellschaftlich am Rande der Bevölkerung stehenden Schichten erreichen und mit den nicht-staatlichen Organisationen der Entwicklungsländer enge Beziehungen unterhalten.

Die Zusammenarbeit mit den Hilfswerken kann zwei Formen an. nehmen : die Regieverträge, im Rahmen derer die DEH den Hilfswerken die Durchführung von Projekten überträgt, die sie selbst identifiziert und ausgearbeitet hat; die Beiträge des Bundes an Projekte der Hilfswerke, wobei diese Unterstützung maximal zwei Drittel des Gesamthaushalts eines Projekts ausmacht. Neben der Finanzierung spezifischer Projekte hat die DEH sechs Hilfswerken - Helvetas, Swissaid, Fastenopfer, Brot für Brüder, Caritas, HEKS - eine Kreditlinie für eine Reihe von Projekten mit einer Laufzeit von ein oder mehreren Jahren eingeräumt.

Die DEH hat den Hilfswerken 1983 einen Gesamtbetrag von 70,5 Mio Fr. zur Verfügung gestellt, die unter rund 40 Organisationen und 167 Entwicklungsprojekten aufgeteilt wurden. Von diesem Betrag hat die DEH mit 27,3 Mio Fr. von den Hilfswerken ausgearbeitete und durchgeführte Aktionen unterstützt und 43,2 Mio Fr. zur Finanzierung der Ausführung von Projekten auf Regiebasis verwendet. Der Umfang der an Intercooperation auf Regiebasis vergebenen Aufträge erreichte 23 Mio Fr.

\section{Zusammenarbeit mit der schweizerischen Privatwirtschaft}

Das Interesse der DEH an einer intensiveren Zusammenarbeit mit der Privatwirtschaft zeigte sich 1983 in der Fortsetzung direkter Kontakte im Hinblick auf den Austausch von Informationen allgemeiner Art und bezüglich der Durchführung spezifischer Aktionen. Die Tatsache, dass die schweizerische Hilfe nicht liefergebunden ist, hindert die DEH nicht daran, oft auf die Dienstleistungen der Privatwirtschaft zurückzugreifen, was in Form der Vergabe von Materialaufträgen an schweizerische Unternehmen oder Regieaufträgen an Ingenieurbüros oder technische Beratungsfirmen erfolgt. Derartige Regieaufträge können sich auf gewisse technische Teilbereiche eines Projektes beziehen (Sachverständigengutachten, Evaluationen, Beratung usw.) oder für die gesamte Durchführung von Projekten (Projekte auf Regiebasis) vergeben werden.

So hat die DEH 1983183 Verträge mit verschiedenen Experten für Kurzzeitmissionen (zum Gesamtbetrag von 1,5 Mio Fr.) abgeschlossen sowie 27 Verträge mit technischen Beratungsfirmen oder Ingenieurbüros für Studien, Evaluationen oder Missionen (Gesamtbetrag 2,5 Mio Fr.).

Zum anderen werden mehrere sehr spezielle Projekte der technischen Zusam- 
menarbeit durch Privatfirmen auf Regiebasis ausgeführt; es handelt sich insbesondere um die Bereiche Hotelwesen, Energie und Milcherzeugung. (Die 1983 neu abgeschlossenen Verträge entsprachen einem Betrag von 5,1 Mio Fr., während sich die Ausgaben auf 2,6 Mio Fr. beliefen).

\section{AUFWENDUNGEN DER DEH FUER 1983 IM BEREICH DER TECHNISCHEN ZUSAMMENARBEIT UND DER FINANZHILFE, NACH DEM EINKOMMENSSTAND DER EMPFAENGERLAENDER AUFGEGLIEDERT}

(in Mio Fr. und Prozent)

\begin{tabular}{lcccc} 
Ländergruppe (1) & $\begin{array}{c}\text { Technische } \\
\text { Zusammenarbeit } \\
\text { Mio Fr. }\end{array}$ & Finanzhilfe & \multicolumn{2}{c}{ Insgesamt } \\
\hline $\begin{array}{l}\text { Am wenigsten entwickelte } \\
\text { Länder (LLDC) }\end{array}$ & 84,6 & 21,0 & 105,6 & 47,5 \\
$\begin{array}{l}\text { Sonstige einkommens- } \\
\text { schwache Länder (BSP } \\
\text { pro Kopf unter 600 \$) }\end{array}$ & 50,2 & 41,2 & 91,4 & 41,1 \\
$\begin{array}{l}\text { Länder mit mittlerem } \\
\text { Einkommen (BSP pro }\end{array}$ & 23,0 & 2,4 & 25,4 & 11,4 \\
$\begin{array}{l}\text { Kopf über 600 \$) } \\
\begin{array}{l}\text { Aufgegliederte Hilfe } \\
\text { insgesamt }\end{array}\end{array}$ & 157,8 & 64,6 & 222,4 & 100,0
\end{tabular}

Regionale und nicht aufgegliederte Projekte (2) INSGESAMT

\begin{tabular}{rrr}
49,7 & 15,1 & 64,8 \\
\hline 207,5 & 79,7 & 287,2
\end{tabular}

1. Die Ländergruppen wurden gemäss der OECD-Definition aufgestellt und beziehen sich auf das Bruttosozialprodukt pro Einwohner vom Jahre 1980.

2. Aktionen, die nicht geographisch aufzugliedern sind, wie Beiträge zu Lehrgängen, Programmen, Globalkredite für Stipendiaten, Finanzierung von Forschungsvorhaben, Unterstützung der Programme gewisser Organisationen usw.

\section{Assoziierte Hilfe und Kofinanzierung}

Die Zusammenarbeit der DEH mit den multilateralen Organisationen zur Durchführung spezifischer Projekte wird assoziierte Hilfe genannt, wenn es sich um Projekte technischer Hilfe handelt, die von den UN-Sonderorganisa- 
tionen verwaltet werden, und Kofinanzierung im Falle von Finanzhilfeprojekten, die von den Entwicklungsbanken oder Entwicklungsfonds durchgeführt werden. In beiden Fällen ist das Verfahren das gleiche : Die DEH wählt ein bestimmtes Projekt aus dem Programm der internationalen Organisation aus. In vielen Fällen nimmt sie direkt an der Ausarbeitung des Projekts und an den Verhandlungen mit dem Empfängerland teil. Die DEH hat die Möglichkeit, auch an Ueberwachungs- oder Evaluierungsmissionen teilzunehmen. Bei Projekten assoziierter Hilfe wird die gesamte externe Projektfinanzierung einschliesslich der Verwaltungskosten von der DEH übernommen. Bei Kofinanzierungen erfolgt die Finanzierung aus verschiedenen Quellen wie schon der Name sagt, d.h. einerseits durch die Entwicklungsbank oder den Entwicklungsfonds, zum anderen durch einen oder mehrere bilaterale oder multilaterale Geber. Diese Form der Zusammenarbeit ermöglicht es, die gute Sachkenntnis der internationalen Organisationen in den Dienst von Projekten zu stellen, die den Zielen der schweizerischen Entwicklungshilfe genau entsprechen. (Die Zahlen betreffend die Projekte der assoziierten Hilfe und der Kofinanzierung sind im statistischen Teil aufgeführt.)

\section{Unterstützung der Arbeiten internationaler Forschungsinstitute durch die $D E H$}

Die DEH leistet seit einigen Jahren Beiträge an gewisse internationale Forschungsinstitute, die für die Entwicklungsländer von Interesse sind. Sie unterstützt insbesondere die zur Beratungsgruppe für internationale Agrarforschung (CGIAR) gehörenden Regionalzentren. Derzeit erhalten zehn Zentren die Hilfe der Schweiz in Form allgemeiner Beiträge oder durch die Finanzierung spezieller Programme. 1983 betrug der dieser Gruppe ausgezahlte Betrag 18 Mio Fr.

Weitere internationale Forschungsinstitute, die die Unterstützung der Schweiz erhalten, sind in der medizinischen Forschung (Infektionskrankheiten, Tuberkulose), dem Umweltschutz oder der Forschung im Agrar- und Forstbereich spezialisiert. Die diesen Instituten 1983 zur Verfügung gestellten Beträge sind in der Tabelle auf Seite 102 aufgeführt.

\section{Unterstützung gewisser schweizerischer Hochschulen durch die DEH und Beteiligung an ihrer Tätigkeit, 1983}

Die DEH nahm 1983 neue interne Leitlinien über die Beziehungen zu den schweizerischen Universitäten und Hochschuleinrichtungen an. Eine der unmittelbaren praktischen Auswirkungen dieses Beschlusses war die Errichtung eines Kleinprogramms zur Förderung der Kontakte zwischen den Hochschulinstitutionen der Schweiz und der Dritten Welt. 1984 wurde beschlossen, das Programm zugunsten der jungen Forschungsarbeiter der DEH zu fördern.

Den Eidgenössischen Technischen Hochschulen in Lausanne und Zürich 


\section{UNTERSTUETZUNG DER ARBEITEN INTERNATIONALER FORSCHUNGSINSTITUTE DURCH DIE DEH, 1983}

(Nettoauszahlungen in Mio Fr.)

\begin{tabular}{|c|c|c|c|}
\hline 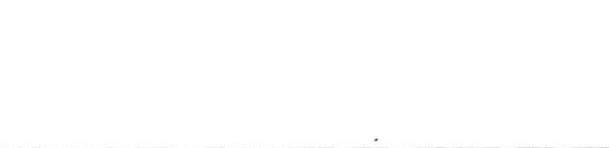 & $\begin{array}{l}\text { Allgemeine } \\
\text { Beiträge }\end{array}$ & $\begin{array}{l}\text { Spezielle } \\
\text { Projekte } \\
\text { und } \\
\text { Programme }\end{array}$ & Insgesamt \\
\hline $\begin{array}{l}\text { Forschungszentren der Beratungsgruppe } \\
\text { für internationale Agrarforschung (CGIAR) }\end{array}$ & 13,5 & 4,8 & 18,3 \\
\hline $\begin{array}{l}\text { Internationales Zentrum für tropische } \\
\text { Landwirtschaft (CIAT), Kolumbien }\end{array}$ & 2,7 & 2,8 & 5,5 \\
\hline $\begin{array}{l}\text { Internationales Zentrum zur Verbesserung } \\
\text { von Mais und Weizen (CIMMYT), Mexiko }\end{array}$ & 3,0 & 0,5 & 3,5 \\
\hline $\begin{array}{l}\text { Internationales Zentrum für Pflanzenbau- } \\
\text { forschung in tropischen Halbtrockengebieten } \\
\text { (ICRISAT), Indien }\end{array}$ & 2,6 & - & 2,6 \\
\hline $\begin{array}{l}\text { Internationales Viehzuchtzentrum für Afrika } \\
\text { (ILCA), Aethiopien }\end{array}$ & 2,1 & - & 2,1 \\
\hline Internationales Kartoffelzentrum (CIP), Peru & 1,0 & 1,1 & 2,1 \\
\hline $\begin{array}{l}\text { Internationales Zentrum für die Erforschung } \\
\text { von Tierkrankheiten (LIRMA), Kenia }\end{array}$ & 1,2 & - & 1,2 \\
\hline $\begin{array}{l}\text { Vereinigung für die Entwicklung des Reisanbaus } \\
\text { in Westafrika (WARDA), Liberia }\end{array}$ & - & 4,0 & 4,0 \\
\hline Sonstige Zentren & 0,8 & - & 1,2 \\
\hline Sonstige Forschungszentren & 1,8 & 4,5 & 6,3 \\
\hline $\begin{array}{l}\text { Internationales Institut für tropische } \\
\text { Landwirtschaft (IITA), Nigeria }\end{array}$ & - & 2,7 & 2,7 \\
\hline $\begin{array}{l}\text { International Centre for Diarrhoeal Diseases } \\
\text { Research (ICDDR), Bangladesh }\end{array}$ & 1,3 & - & 1,3 \\
\hline $\begin{array}{l}\text { Programm für Forschung und Ausbildung } \\
\text { über Umweltfragen (ENDA), Senegal }\end{array}$ & - & 0,8 & 0,8 \\
\hline Internationale Tuberkulose-Vereinigung (UICT) & - & 0,7 & 0,7 \\
\hline $\begin{array}{l}\text { International Council for Research in } \\
\text { Agro-Forestry (ICRAF), Kenia }\end{array}$ & 0,5 & - & 0,5 \\
\hline Sonstige Institute & - & 0,3 & 0,3 \\
\hline INSGESAMT & 15,3 & 9,3 & 24,6 \\
\hline
\end{tabular}


wurde für ihre jeweiligen Nachdiplomkurse über Entwicklungsfragen weiterhin Unterstützung gewährt. Auch wurden Kontakte aufgenommen, um folgenden Fachinstituten der ETH Aufträge und technische Unterstützung zukommen zu lassen : Institut für Ernährungswissenschaft, Institut für Tierproduktion, Institut für Agrarwirtschaft, Institut für Wasserwirtschaft, Wasserreinigung und Wasserschutz.

\subsection{Multilaterale Entwicklungszusammenarbeit}

Die multilaterale Entwicklungszusammenarbeit ist Bestandteil der internationalen Hilfsaktionen für die Dritte Welt, an denen auch die Schweiz teilnimmt.

Die multilaterale Hilfe kann nach Meinung des Bundesrates Funktionen ausüben, die die bilaterale Hilfe ihrerseits nicht übernehmen kann. Insisesondere ist die multilaterale Hilfe ein konstanter Prozess, während die bilaterale Hilfe oft von konjunkturellen und kurzfristigen Faktoren abhängig ist.

Die Institutionen der multilateralen Entwicklungszusammenarbeit sind aufgrund ihrer Grösse und ihrer Infrastruktur häufig in der Lage, neuauftretende Probleme zu meistern und eine Koordinierungsrolle auszuüben. Gewisse Vorhaben machen Massnahmen in sehr grossem Umfang erforderlich.

Die spezialisierten Organisationen haben die nötige Erfahrung und Sachkenntnis in ihrem jeweiligen Bereich. Der Umfang ihres Verwaltungsapparates und das Ausmass ihrer Projekte ermöglichen es ihnen, Privatkapital für Kofinanzierungen zu mobilisieren.

Die Organisationen der multilateralen Entwicklungszusammenarbeit spielen bei der Festlegung nationaler und internationaler Entwicklungsstrategien eine koordinierende Rolle zwischen den Ländern. Ein weiterer Vorteil für die Entwicklungsländer ist, dass die multilaterale Hilfe nicht gebunden ist.

Was die Zuständigkeiten innerhalb der eidgenössischen Verwaltung betrifft, so ist die DEH prioritär für Fragen der technischen Hilfe verantwortlich und hat mit dem Bundesamt für Aussenwirtschaft (BAWI) zusammen die Verantwortung für die finanzielle Hilfe. In Bezug auf die internationalen Organisationen, die in den Kompetenzbereich anderer Stellen oder Departemente fallen, hat die DEH ihre Meinung zu Fragen der Entwicklungspolitik abzugeben.

Seit 1982 wendet die Schweiz im Rahmen der internationalen Finanzhilfe die Zahlungsweise der "promisory notes" an. Hier handelt es sich um Beiträge, die in Form von unwiderruflichen Schuldanerkennungen erfolgen, jedoch erst zum Zeitpunkt der tatsächlichen Auszahlungen gebucht werden, da diese sich über einen Zeitraum bis zu zehn Jahren erstrecken können. Die Uebergangsphase könnte sich noch über einige Jahre hinziehen. 
In der Folge sollten die bilateralen und multilateralen Beiträge ihr Gleichgewicht in dem bekannten Verhältnis von rund $\mathbf{4 0} \%$ für die multilaterale Hilfe und $60 \%$ für die bilaterale Hilfe wiedererlangen.

Wie in den vorhergehenden Jahren ging der grösste Beitrag der Schweiz im Bereich der multilateralen Zusammenarbeit an das Entwicklungsprogramm der Vereinten Nationen (PNUD). Das PNUD spielt eine wichtige Rolle in der internationalen Entwicklungshilfe. Es stellt jedem Entwicklungsland einen Rahmenkredit bereit, der die Durchführung eines den Bedürfnissen der Hilfeempfänger bestmöglich entsprechenden Programms gestattet. Rund $80 \%$ der Mittel des PNUD sind für die ärmsten Länder bestimmt. Der Schweizer Anteil am PNUD entspricht 2,5\% der Gesamtbeiträge.

Ferner unterstützt die Schweiz folgende Organisationen :

Fonds für die am wenigsten entwickelten Länder (FPMA). Es handelt sich um einen im Rahmen des PNUD errichteten Sonderfonds, dessen Eingliederung in das ordentliche PNUD-Programm eine optimale Nutzung der Mittel gewährleistet. 1983 hat die Schweiz einen ordentlichen Beitrag von 4,84 Mio Fr. in den Fonds für die am wenigsten entwickelten Länder eingezahlt. Im gleichen Jahr hat der Fonds von der Schweiz einen Sonderbeitrag von 5 Mio Fr. im Rahmen einer kurzfristigen finanziellen Unterstützung zugunsten des PNUD erhalten.

Ausrüstungsfonds der Vereinten Nationen (FENU). Dieser Fonds, der seit 1966 besteht, kommt heutzutage fast ausschliesslich den am wenigsten entwickelten Ländern zugute. Er hat insbesondere die Aufgabe, diesen Ländern eine Ausrüstungshilfe für kleine und mittlere Projekte zukommen zu lassen. Er vergewissert sich, dass die von ihm finanzierten Projekte zur Befriedigung der Lebensbedürfnisse der ärmsten Bevölkerungsgruppen beitragen. Die Mittel des FENU werden von einer begrenzten Anzahl von Ländern aufgebracht, zu denen auch die Schweiz zählt.

Fonds der Vereinten Nationen für Bevölkerungsfragen (FNUAP). Der FNUAP ist derzeit die wichtigste internationale Institution, die Aktivitäten betreffend Bevölkerungsfragen finanziert und durchführt. Seine Finanzierung wird durch freiwillige Jahresbeiträge der Mitgliedsländer gewährleistet. Die Ausführung seiner Projekte wird im allgemeinen den Sonderorganisationen der Vereinten Nationen, wie WHO, UNICEF, UNESCO, oder den Regierungen der betreffenden Länder selbst übertragen.

Kinderhilfswerk der Vereinten Nationen (UNICEF). Die Schweiz hat von Anfang an direkt an der Tätigkeit des UNICEF teilgenommen und hat seit der Errichtung des Hilfswerksständig einen Sitz im Verwaltungsrat inne.

Weltgesundheitsorganisation (WHO). Im Rahmen der 36. Generalversammlung der Organisation nahm die Schweiz im besonderen an den Arbeiten zur Aenderung und Ergänzung der Strategie "Gesundheit für alle bis zum Jahr 2000" teil. Ferner hat die DEH 1983 zwei Aktionen der WHO, nämlich das 
Programm zur Bekämpfung der Diarrhoekrankheiten und das Forschungsund Ausbildungsprogramm zur Bekämpfung der Tropenkrankheiten, mit einem Betrag von insgesamt 2,25 Mio Fr. unterstützt.

\section{Organisation der Vereinten Nationen für Erziehung, Wissenschaft und} Kultur (UNESCO). Die DEH hat sich 1983 verpflichtet, ein Projekt zur Ausbildung auf dem Gebiet des Fernmeldewesens in Simbabwe, in Höhe von 2,9 Mio Fr., im Rahmen der assoziierten Hilfe zu unterstützen.

\section{Internationaler Fonds für Landwirtschaftliche Entwicklung (FIDA). Der} FIDA wurde 1977 mit dem Ziel geschaffen, zusätzliche Finanzmittel für die Entwicklungsländer zu mobilisieren, um ihnen zu helfen, ihre Lebensmittelproduktion zu steigern und die Ernährungsgrundlage ihrer Bevölkerung zu verbessern.

Die Mitglieder des FIDA sind in drei Gruppen aufgeteilt, welche unabhängig von der Beitragshöhe oder der Mitgliederzahl über die gleiche Anzahl Stimmen verfügen : Industrieländer, OPEC-Staaten, Entwicklungsländer.

Die Finanzierung des FIDA erfolgt hauptsächlich durch die Mitglieder der ersten beiden Gruppen, im derzeitigen Verhältnis von 58 \% für die Industrieländer und 42 \% für die erdölexportierenden Länder.

Die Lage des Fonds ist zur Zeit relativ schwierig, nachdem die Vereinigten Staaten im Dezember 1983 angekündigt hatten, dass sie anstatt ihres Beitrags in Höhe von 183 Mio Dollar, den sie bis Ende 1983 zu leisten hatten, nur die Hälfte, nämlich 90 Mio Dollar einzahlen würden. So haben in der Gruppe I nur die Schweiz, Australien, die Niederlande, Oesterreich, Italien und die skandinavischen Länder ihren Beitrag voll eingezahlt, während die anderen Länder die endgültige Entscheidung der USA abwarten. Die Haltung der Vereinigten Staaten könnte sich aus deren Enttäuschung gegenüber der Tatsache erklären, dass die OPEC-Länder einen kleineren Anteil der Aufwendungen als vorgesehen übernommen haben.

Anlässlich der Jahreskonferenz der Vereinten Nationen, die im Herbst 1983 in New York stattfand, gab der ständige Beobachter der Schweiz bei den Vereinten Nationen, Botschafterin Francesca Pommetta bekannt, welche Hauptbeiträge die Schweiz für verschiedene Institutionen, Sonderfonds und Sonderprogramme der Vereinten Nationen bereitzustellen beabsichtigt.

Den höchsten Beitrag der Schweiz, nämlich 40 Mio Fr., erhält wie gewöhnlich das Entwicklungsprogramm der Vereinten Nationen (PNUD), welches das UN-Zentralorgan für die Finanzierung und Koordinierung der technischen Hilfe ist.

Das Kinderhilfswerk der Vereinten Nationen (UNICEF) erhält 9 Mio Fr. als allgemeinen Beitrag. Ferner beteiligt sich die Schweiz an mehreren spezifischen Projekten.

Weitere bedeutende Beiträge sind für folgende Sonderfonds und -programme vorgesehen : 5,3 Mio Fr. für den Fonds für die am wenigsten entwickelten 
Länder; 4,235 Mio Fr. für den Ausrüstungsfonds; 4 Mio Fr. für den Fonds für Bevölkerungsfragen (FNUAP) und 0,4 Mio Fr. für das Freiwilligenprogramm.

Des weiteren hat die Schweiz für zwei Jahre eine Summe von 4,5 Mio Fr. für die Finanzierung von Projekten des Fonds für die Industrieentwicklung (FNUDI) bereitgestellt und wird für die Jahre 1983-1986 einen Betrag von 4 Mio Fr. für das gemeinsame Energieprogramm des PNUD und der Weltbank leisten.

Als Mitglied der leitenden Organe des PNUD und des UNICEF nimmt die Schweiz aktiv an der Gestaltung und Kontrolle der Tätigkeiten dieser Organisationen teil.

\section{Regionale Entwicklungsbanken und Entwicklungsfonds}

Der Bundesrat hat beschlossen, sich an der 6. Kapitalaufstockung der Interamerikanischen Entwicklungsbank zu beteiligen und den Anteil der Schweiz auf 65 Mio Fr. festzulegen. 4,5\% dieses Betrages (2,96 Mio Fr.) werden effektiv eingezahlt. Der Rest dient als Garantiekapital. Ferner beteiligt sich die Schweiz an der 6. Wiederauffüllung des der Bank angeschlossenen Fonds für Sonderaktionen, in Form eines Geschenks von 11,5 Mio Fr.

Die in vier Jahrestranchen (1983-1986) zahlbaren Beträge werden aus bereits bestehenden Rahmenkrediten finanziert. Das Parlament hatte seinerzeit die vorgesehene Verwendung dieser Kredite bereits gebilligt.

Die Schweiz ist seit 1979 Mitglied der Interamerikanischen Entwicklungsbank. Die Unterstützung der Länder der Dritten Welt im Rahmen multilateraler Institutionen ist Bestandteil der schweizerischen Entwicklungszusammenarbeit.

Die Entwicklungsbank der Zentralafrikanischen Staaten (BDEAC) und die Schweizer Regierung haben am 17. Februar 1984 ein Abkommen unterzeichnet, das eine Finanzhilfe in Höhe von 6,8 Mio Fr. für diese Institution vorsieht. Die Hilfe ist dazu bestimmt, die Lebensbedingungen der benachteiligten Bevölkerungen und Gebiete Zentralafrikas zu verbessern.

Die BDEAC wurde 1975 von den Staaten Kamerun, Kongo, Gabon, Zentralafrikanische Republik und Tschad als sogenannte "intra-regionale" Entwicklungsbank gegründet. Ihr Ziel ist, die Zusammenarbeit zu fördern, um die regionalen Unterschiede auszugleichen und insbesondere den Rückstand im landwirtschaftlichen Sektor aufzuholen.

Seit 1980 war die DEH bestrebt, die BDEAC bei der Errichtung einer Abteilung für ländliche Entwicklung zu unterstützen. Heute ist diese Abteilung bereits in Betrieb. Sie ist imstande, Projekte der ländlichen Entwicklung in der Region zu identifizieren, auszuarbeiten und zu betreuen, wobei sie gegebenenfalls die Dienste technischer Berater (Agronomen, Bauingenieure, Volkswirte, usw.) in Anspruch nimmt. 
Die finanzielle Unterstützung der BDEAC durch die Schweiz ist eine Ergänzung der dieser Institution bereits gewährten Hilfe. Sie umfasst zwei Programme : Einerseits einen nicht rückzahlbaren Beitrag von $800.000 \mathrm{Fr}$., der dazu bestimmt ist, die Kapazität der Bank im Bereich der ländlichen Entwicklung nochauszubauen. (Die schweizerischen Mittel werden für die Finanzierung vorbereitender Projektstudien und Beratungsmissionen eingesetzt.) Andererseits ein Darlehen von 6 Mio Fr. zu Vorzugsbedingungen für die Kofinanzierung von Projekten ländlicher Entwicklung in Zusammenarbeit mit der Bank.

Die Zusagen der schweizerischen Finanzhilfe werden innerhalb einer Frist von zwei Jahren erteilt. Die DEH wird im Rahmen des mit der Zentralafrikanischen Entwicklungsbank unterzeichneten Abkommens die Zuteilung und Verwendung der schweizerischen Mittel kontrollieren.

\section{HUMANITAERE HILFE}

Die Gesamtaufwendungen für die humanitäre Hilfe der Eidgenossenschaft beliefen sich 1983 auf 124,94 Mio Fr. In diesem Betrag sind die Aufwendungen für Länder, die offiziell nicht als Entwicklungsländer angesehen werden, inbegriffen. So wurden 1983 1,6 Mio Fr. (1982 : 3,9 Mio Fr.) ausserhalb der Dritten Welt eingesetzt.

Neben den Aktionen im Rahmen von Naturkatastrophen und bewaffneten Konflikten steigt der Anteil der Flüchtlings- und Vertriebenenhilfe an.

Die humanitäre Hilfe wird in Zusammenarbeit mit den nationalen und internationalen Hilfswerken sowie mit dem schweizerischen Freiwilligenkorps für Katastrophenhilfe im Ausland verwaltet.

Im Jahre 1983 hat sich der Tätigkeitsbereich des Freiwilligenkorps für Katastrophenhilfe erweitert. 145 Freiwillige nahmen an den Hilfsaktionen teil. Ende des Jahres verfügte das Katastrophenhilfekorps für seine Aktionen über einen Materialbestand im Werte von 4,5 Mio Fr., während sich die Ausgaben auf rund 2,2 Mio Fr., d.h. etwa ein Viertel der Gesamtaufwendungen, beliefen.

Es wurden Untersuchungen im Hinblick auf den Erwerb neuer mobiler Hydraulikausrüstungen sowie die Verbesserung hochwandiger Familienzelte mit Doppeldach zu Ende geführt.

Das Katastrophenhilfskorps war 1983 in Aequatorialguinea, Ghana, Guinea (Conakry), Italien, Kolumbien, Nordjemen, Obervolta, Peru, Südjemen, im Tschad und in der Türkei im Einsatz.

Die Aufteilung der humanitären Hilfe nach Gebieten ergibt folgende Anteile: 
Afrika 53,5\%; Mittlerer Osten 13,2 \%; Asien 18,1\%; Lateinamerika 9,1\%; Europa $1,1 \%$; Verschiedenes $5 \%$.

Im Bereich der Nahrungsmittelhilfe wurden von der Schweiz 198336.400 Tonnen Nahrungsmittel im Wert von insgesamt 55,6 Mio Fr. (Finanzierung, Transport und Auslieferung inbegriffen) geliefert. Dieser Betrag entsprach $0,4 \%$ der weltweiten Nahrungsmittelhilfe.

$85 \%$ dieser Hilfe waren für Flüchtlinge und Notleidende bestimmt; $60 \%$ wurden in Afrika verteilt. 51 \% der Getreidehilfe kam aus den Entwicklungsländern selbst (vor allem Afrika).

Indien, der Tschad, Aethiopien, der Libanon, Malawi, Brasilien, sowie die Palästinaflüchtlinge im Nahen Osten und die Flüchtlinge aus dem Ogadengebiet in Somalia waren die Hauptempfänger für die Hilfe an Milchprodukten, während die Getreidehilfe vor allem den Palästinaflüchtlingen sowie den Ländern Aethiopien, Ghana und Rwanda zugute kam.

2,7 Mio Fr. wurden schweizerischen Hilfswerken für die Durchführung von humanitären Nothilfeaktionen in 19 Ländern zur Verfügung gestellt. Hilfsaktionen in Höhe von 1 Mio Fr. wurden ebenfalls mit der Unterstützung von neun schweizerischen Vertretungen durchgeführt.

Die Schweiz hat angekündigt, dass sie dem HCR 1984 einen ordentlichen Beitrag von 4,5 Mio Fr. zukommen lassen und im Rahmen der finanziellen Möglichkeiten ausserordentliche Beiträge für Sonderprogramme leisten werde. Ferner werde sie dem HCR Experten des schweizerischen Freiwilligenkorps für Katastrophenhilfe für zeitlich begrenzte Missionen zur Verfügung stellen. Diese Beiträge werden durch den Rahmenkredit für internationale humanitäre Hilfe finanziert. Die Schweiz ist Mitglied des Exekutivkomitees des HCR.

Der Bundesrat hat beschlossen, die Nahrungsmittelhilfe (Nothilfeprogramm) für 1984 um 5 Mio Fr. zugunsten Afrikas zu erhöhen. Dieser ausserordentliche Beitrag wird ebenfalls aus dem Rahmenkredit für internationale humanitäre Hilfe finanziert. Die in Afrika derzeit am meisten bedrohten Länder sind : Aethiopien, Ghana, Guinea-Bissau, Kap Verde und Mosambik.

Im vergangenen Jahr belief sich die Getreidehilfe der Schweiz auf 24.130 Tonnen (was dem Gegenwert von 29.405 Tonnen Weizen entspricht). Sie umfasste folgende Produkte : 10.500 Tonnen schweizerisches Brotmehl, sowie 13.630 Tonnen Mais, Maismehl und Sorgho aus Argentinien, Kenia und Rwanda.

Die Ware wurde für Hilfsaktionen der Caritas, des IKRK, des Welternährungsprogramms und der UNRWA nach Bolivien, Aethiopien, Ghana, Mali, Peru, Rwanda und in den Mittleren Osten gebracht. Ferner hat die Regierung von Kap Verde eine Zuteilung erhalten.

Die Getreidehilfe der Schweiz erfolgt im Rahmen des internationalen Nahrungshilfeabkommens im Zusammenhang mit dem internationalen Getreide- 
abkommen, dem sie angehört. Die Mindestverpflichtung beläuft sich auf den Gegenwert von 27.000 Tonnen Weizen im Jahr. Diese Menge entspricht $0,35 \%$ der Gesamtverpflichtungen der 12 Mitglieder in Höhe von 7,6 Mio Tonnen.

Im Rahmen dieser internationalen humanitären Hilfe ist vorgesehen, 1984 hauptsächlich im Rahmen des Nothilfe- und Flüchtlingshilfeprogramms 27.000 Tonnen Getreide an Entwicklungsländer zu liefern. Der für diesen Zweck zur Verfügung stehende Kredit beläuft sich laut Budget auf 17,85 Mio Fr.

\section{STIPENDIEN}

Die Aenderung des Bundeserlasses vom 19. Dezember 1980 gewährleistete weiterhin die Finanzierung des vom Bundesamt für Kulturpflege des EDI verwalteten Hochschulstipendienprogramms. Die Mehrzahl der in der Schweiz studierenden Stipendiaten kommt aus Ländern der Dritten Welt (180 Studenten). Die Aufwendungen des EDI beliefen sich auf 2,5 Mio Fr.

Zusätzlich wurden 1983 im Rahmen der bilateralen technischen Zusammenarbeit (einschliesslich der assoziierten Hilfe) 284 Stipendiaten in der Schweiz ausgebildet. Diese Fortbildungslehrgänge sind für Personen der Dritten Welt bestimmt, die bereits Berufserfahrung haben. Die diesbezüglichen Aufwendungen betrugen rund 3 Mio Fr. $65 \%$ der Stipendiaten kamen aus Afrika und $19 \%$ aus Asien. Die meisten von innen erhielten eine Ausbildung im technisch-gewerblichen Bereich (17\%), im Dienstleistungssektor (Post- und Fernmeldenwesen, Hotelgewerbe, öffentliche Gesundheit : $13 \%, 10 \%$, $10 \%)$, sowie in den Bereichen Versicherungen (6\%), Banken (4\%), oder öffentliche Verwaltung $(6 \%)$.

\section{AUFWENDUNGEN VON KANTONEN UND GEMEINDEN}

Diese öffentlichen Körperschaften erbrachten 1983 Leistungen in Höhe von insgesamt 8,42 Mio Fr., die rund 1,5\% der gesamten öffentlichen Entwicklungshilfe der Schweiz entsprachen.

22 Kantonen brachten rund 4 Mio Fr. und 140 Gemeinden 4,5 Mio Fr. auf. Rund $80 \%$ dieser Beiträge entfallen auf die Entwicklungszusammenarbeit und $20 \%$ auf die humanitäre Hilfe.

Von den 4 Mio Fr., die von den Kantonen aufgebracht worden sind, wurden 
$73 \%$ von den vier Kantonen Genf, Basel-Stadt, Zug und Zürich beigetragen. Von den 4,46 Mio Fr., die den Leistungen der Gemeinden für die öffentliche Entwicklungshilfe entsprechen, wurden über $77 \%$ von 65 Gemeinden in den Kantonen Zürich, Genf und Bern erbracht.

\section{PRIVATE ENTWICKLUNGSZUSAMMENARBEIT}

Die private Entwicklungszusammenarbeit ist eine eigenständige Leistung der verschiedenen Hilfsorganisationen, Missionswerke und anderer, privater Organisationen der Schweiz. Zum Teil sind ihre Aktivitäten mit denen des Bundes finanziell und organisatorisch verbunden, insbesondere werden gewisse private Leistungen durch Bundesmittel ergänzt.

1983 konnten die privaten Hilfsorganisationen religiösen und nicht-religiösen Charakters 125,17 Mio Fr. aus Spendengeldern für ihre Entwicklungshilfetätigkeit ausgeben. 1982 belief sich dieser Betrag auf 105,4 Mio Fr. Diese Tätigkeit untergliedert sich in die eigentliche Entwicklungshilfe, für welche 103,8 Mio Fr. zur Verfügung standen und die humanitäre Hilfe, für welche 21,78 Mio Fr. zur Verfügung standen. Die private Entwicklungshilfe wurde durch Bundesmittel in der Höhe von 373,72 Mio SFr. ergänzt, die private humanitäre Hilfe durch Bundesmittel in der Höhe von 123,36 Mio Fr.

Am meisten Mittel konnten von den Hilfsorganisationen Caritas, HEKS, Fastenopfer, Schweiz. Rotes Kreuz und von den Missionsgesellschaften "Institut Ingenbohl" und "Bethlehem" aufgebracht werden. Am meisten Bundesbeiträge erhielten Intercooperation und Helvetas.

Die Leistungen der privaten Hilfsorganisationen beschränken sich nicht nur auf technische Zusammenarbeit und humanitäre Hilfe. Zahlreich sind ihre Aktivitäten in der Schweiz selbst. Einzelne Organisationen führen gar keine Projekte in Entwicklungsländern durch. Sie legen ihre Hauptaufgabe auf die Information der Bevölkerung über Entwicklungsfragen.

Die bedeutendste Aktivität, mit der die Hilfswerke im Berichtsjahr an die Oeffentlichkeit gelangten, war die Aktion "Für eine Welt ohne Hunger" der Hilfswerke Swissaid, Fastenopfer, Brot für Brüder, Helvetas, HEKS, Caritas, SAH und SRK. Im Mai 1984 riefen diese zusammen mit der "Glückskette" von Radio und Fernsehen zu dieser Informations- und Sammelkampagne auf. Bis Mitte 1984 wurden zu diesem Zweck über 10 Mio Fr. gespendet. Die Aktion wird bis Ende 1984 fortgeführt und hat zum Ziel, über die verschiedenen Ursachen des Hungers zu informieren, zusätzliche kurzfristige Nothilfemassnahmen in den vom Hunger am härtesten betroffenen Ländern Afrikas und Lateinamerikas zu ermöglichen und die längerfristige Projektarbeit der Hilfswerke zu verstärken. 
Die in der letztjährigen Aktion "Entwicklungshilfe ist eine Ueberlebensfrage" zusammengeschlossenen Hilfswerke, welche dieser Aktion durch eine Petition besonderes Gewicht gaben, wandten sich im Zusammenhang mit der Meinungsumfrage "Entwicklungspolitik" (vgl. Jahrbuch 1984, Untersuchungen und stellungnahmen) im Mai 1984 erneut an die Oeffentlichkeit. Sie werten die Ergebnisse dieser Umfrage, welche von der DEH und Hilfswerken gemeinsam in Auftrag gegeben wurde, als ermutigend für ihre Arbeit, sehen sich aber weiterhin einem Informationsmangel unter der schweizerischen Bevölkerung über Entwicklungsländer und die Entwicklungszusammenarbeit gegenüber.

Gleichzeitig forderten diese Hilfswerke die Parlamentarier auf, auf die vorgesehenen Sparmassnahmen und Kürzungen bei der Entwicklungshilfe zu verzichten. Weiter wollen sie die künftige Entwicklungshilfe der Schweiz vermehrt an folgenden Zielen orientiert sehen :

- Ausrichtung der Hilfe auf die Grundbedürfnisse der ärmsten Bevölkerungsschichten und auf nichtstaatliche Organisationen als Partner für Projekte,

- vermehrte Förderung von Gewerbe und Kleinstbetrieben zur Lösung des Beschäftigtenproblems,

- Massnahmen zum Schutz der Umwelt und die Bekämpfung der Bevölkerungsexplosion.

Die zielorientierte Entwicklungszusammenarbeit soll im übrigen durch ein nationales Forschungsprogramm, welches die Entwicklungsstrategien für die 90er Jahre erforschen soll, ergänzt werden.

Zur Diskussion der komplexen Beziehungen Schweiz - Dritte Welt ist im Februar 1984 die "Studiengesellschaft Schweiz - Dritte Welt" gegründet worden. Präsident dieser Studiengesellschaft ist E.A. Brugger. Ihre Mitglieder aus Wissenschaft, Wirtschaft und Entwicklungsorganisationen wollen gemeinsam in themenbezogenen Studiengruppen prioritäre Fragenkreise dieser Beziehungen bearbeiten.

\section{Quellen}

Rapport de gestion du Conseil fédéral, 1983.

Rapport annuel de la DDA, 1983.

Message concernant la continuation de la coopération technique et de l'aide financière en faveur des pays en développement, 19 mars 1984.

Bulletin officiel de l'Assemblée fédérale.

Plan financier 1984-85 (DFF).

Actualités i3m (Service d'information Tiers Monde, Lausanne).

EVD-Pressemitteilungen, 20.7.83, 16.5.84.

EVD-Pressemitteilung, 16.1.84.

Pressecommuniqué “Für eine Welt ohne Hunger”, 21.6.84.

Swissaid, Schweiz, Hilfe für Entwicklungsländer, Bern 1984.

NZZ, 17.2., 14.4., 23.5.84.

Tages-Anzeiger, 23.5.84. 